\title{
Equivariant Versal Deformations of Semistable Curves
}

\author{
Jarod Alper \& ANDreW Kresch
}

\begin{abstract}
We prove that given any $n$-pointed prestable curve $C$ of genus $g$ with linearly reductive automorphism group $\operatorname{Aut}(C)$, there exists an $\operatorname{Aut}(C)$-equivariant miniversal deformation of $C$ over an affine variety $W$. In other words, we prove that the algebraic stack $\mathfrak{M}_{g, n}$ parameterizing $n$-pointed prestable curves of genus $g$ has an étale neighborhood of $[C]$ isomorphic to the quotient stack $[W / \operatorname{Aut}(C)]$.
\end{abstract}

\section{Introduction}

A fundamental question in algebraic geometry is to understand the relationship between arbitrary algebraic stacks and quotient stacks. While not every algebraic stack is a quotient stack ([7] and [11]), it is natural to conjecture that every algebraic stack is étale locally a quotient stack around a point with linearly reductive stabilizer. Precisely, we formulate the conjecture as follows.

CONJECTURE 1.1. Let $X$ be an algebraic stack locally of finite type over an algebraically closed field $k$ with separated and quasi-compact diagonal such that $X$ has affine stabilizer groups at all closed points. Suppose $x \in X(k)$ has a linearly reductive stabilizer group scheme $G_{x}$. Then there exists an affine scheme $W$ over $k$ with an action of $G_{x}, a$ k-point $w \in W$, and an étale, representable morphism

$$
f:\left[W / G_{x}\right] \rightarrow X
$$

such that $f(w)=x$ and $f$ induces an isomorphism of stabilizer groups at $w$.

Conjecture 1.1 after replacing $W$ with an algebraic space is a particular case of the conjecture stated in [3]. Similar questions were raised in [5, §5] and [16, §2].

This conjecture implies that étale-local properties of general algebraic stacks (satisfying the hypotheses of Conjecture 1.1) can be inferred from properties of algebraic stacks of the form $[\operatorname{Spec}(A) / G]$ with $G$ linearly reductive. Such quotient stacks are particularly well understood; in particular, many geometric properties of $[\operatorname{Spec}(A) / G]$ can be related to properties of the GIT quotient $\operatorname{Spec}\left(A^{G}\right)$. Additionally, as suggested by Rydh, it is possible to attach to an algebraic stack $X$ satisfying Conjecture 1.1 at a point $x \in X(k)$ a Henselian localization $\mathcal{O}_{X, x}^{h}$ that is a comodule algebra over the Hopf algebra of $G_{x}$ such that $\left[\operatorname{Spec}\left(\mathcal{O}_{X, x}^{h}\right) / G_{x}\right] \rightarrow X$ satisfies analogous properties to the usual Henselization $\operatorname{Spec}\left(\mathcal{O}_{W, w}^{h}\right) \rightarrow W$.

Received March 25, 2014. Revision received January 27, 2016.

The first author was partially supported by the Alexander von Humboldt Foundation. The second author was partially supported by the Swiss National Science Foundation. 
Conjecture 1.1 is known to have a positive answer if $X$ has quasi-finite diagonal (e.g., $X$ is a Deligne-Mumford stack) or if $X=[U / G]$ where $U$ is a normal scheme and $G$ is a linear algebraic group (see Sections 3.2-3.3). The purpose of this article is to verify Conjecture 1.1 in an interesting and natural moduli problem, which does not fall into one of the cases mentioned. Let $\mathfrak{M}_{g, n}$ be the moduli stack of prestable curves (proper flat families of connected nodal curves) of genus $g$ with $n$ marked points.

THEOREM 1.2. Conjecture 1.1 holds for $\mathfrak{M}_{g, n}$ for all $g, n \geq 0$.

This theorem implies that given any $n$-pointed prestable curve $C$ of genus $g$ with linearly reductive automorphism group $\operatorname{Aut}(C)$, there exists an affine variety $W$ with an action of $\operatorname{Aut}(C)$ fixing a point $w \in W$ and a miniversal deformation $\mathcal{C} \rightarrow W$ of $C \cong \mathcal{C}_{w}$ such that there is an action of $\operatorname{Aut}(C)$ on the total family $\mathcal{C}$ compatible with the action on $W$ and restricting to the natural action of $\operatorname{Aut}(C)$ on $\mathcal{C}_{w}$.

Let $\mathfrak{M}_{g, n}^{\text {ss }} \subset \mathfrak{M}_{g, n}$ be the open substack consisting of semistable curves (i.e., pointed curves $\left(C,\left\{p_{i}\right\}_{i=1}^{n}\right)$ such that $\omega_{C}\left(\sum_{i} p_{i}\right)$ has nonnegative degree on every component, where $\omega_{C}$ is the dualizing sheaf). Since a prestable curve with a linearly reductive automorphism group, which is not a 0 -pointed smooth curve of genus 0 , is semistable, Theorem 1.2 reduces to proving that $\mathfrak{M}_{g, n}^{\mathrm{ss}}$ satisfies Conjecture 1.1 .

The algebraic stack $\mathfrak{M}_{g, n}^{\text {ss }}$ has particularly exotic properties in connection to Conjecture 1.1. For instance, $\mathfrak{M}_{g, n}^{\mathrm{ss}}$ has a finite-type open substack that is not a global quotient stack, does not have quasi-affine diagonal, and does not admit a good moduli space (see Section 2.2). For these reasons, $\mathfrak{M}_{g, n}^{\text {ss }}$ serves as a natural test case for Conjecture 1.1.

Finally, the technique employed to prove Theorem 1.2, based on stacks of log structures, reveals features of the stack of semistable curves that may be of independent interest. For instance, Lemma 4.3 gives a description of the local structure of the stack of semistable curves near a curve whose stabilization has just one node, in terms of a particular zero-dimensional smooth algebraic stack, which has appeared in many settings in algebraic geometry and has been studied in detail by Abramovich, Cadman, Fantechi, and Wise [1]. In particular, this provides a concrete description of the fiber of the stabilization morphism over a stable curve with one node.

The proof of Theorem 1.2 proceeds by a sequence of reductions. In Section 2, we show that it suffices to prove that $\mathfrak{M}_{g}^{\text {ss }}$ satisfies Conjecture 1.1 for all $g \geq 0$ (Proposition 2.1); in that section, we also exhibit some exotic properties of the stack $\mathfrak{M}_{g}^{\text {ss }}$. In Section 3, we state a result (Proposition 3.10) that reduces Conjecture 1.1 for $\mathfrak{M}_{g}^{\text {ss }}$ to showing the existence of particular kinds of finite covers of étale neighborhoods. Just this is enough to verify Conjecture 1.1 in the special case (Theorem 4.2) of a pair of smooth curves of distinct positive genera joined by a chain of rational curves (in Section 4). After presenting some stacks generalities in Section 5 and a local construction around a strictly semistable curve in 
Section 6, the next task will be to formulate and prove, in Section 7, a structure result (Proposition 7.1) for the moduli of semistable curves with stabilization contracting a single chain of rational curves to a node. Finally, Theorem 1.2 is proved in Section 8.

\section{Conventions}

In this paper we work with general algebraic stacks (not required to have separated or quasi-compact diagonal) as defined in [21]. An algebraic stack possessing an étale cover by a scheme is called a Deligne-Mumford stack. Sheaves of monoids arise in the treatment of log structures; all (sheaves of) monoids are commutative.

\section{Stable and Semistable Curves}

Fix an algebraically closed field $k$ and $g \geq 2$. Let $\mathfrak{M}_{g, n}$ be the moduli stack of prestable curves (i.e., nodal, connected, proper curves) of genus $g$ with $n$ marked points, with stabilization morphism

$$
\text { st: } \mathfrak{M}_{g, n} \rightarrow \overline{\mathcal{M}}_{g, n},
$$

which is flat, to the stack $\overline{\mathcal{M}}_{g, n}$ of stable curves of genus $g$ with $n$ marked points. The algebraic stack $\mathfrak{M}_{g, n}$ is quasi-separated and locally of finite type over $k$. Let $\mathfrak{M}_{g}^{\text {ss }} \subset \mathfrak{M}_{g}$ denote the locus of semistable curves, that is, curves whose dualizing sheaf has nonnegative multidegree. (A similar definition can also be made for $\mathfrak{M}_{g, n}^{\mathrm{ss}} \subset \mathfrak{M}_{g, n}$.) We will also consider $\mathfrak{M}_{g}^{\mathrm{qs}} \subset \mathfrak{M}_{g}^{\mathrm{ss}}$, the locus of quasi-stable curves, that is, semistable curves where the exceptional components (smooth rational components on which the degree of the dualizing sheaf is zero) are pairwise disjoint.

We recall, in the context of algebraic stacks of finite presentation over $k$, a global quotient stack, or just quotient stack, is a stack quotient $[U / G]$ for the action of a linear algebraic group $G$ on a finite-type algebraic space $U$. Choosing a faithful representation $G \rightarrow \mathrm{GL}_{n}$, we have

$$
[U / G] \cong\left[U \times{ }^{G} \mathrm{GL}_{n} / \mathrm{GL}_{n}\right],
$$

where $U \times{ }^{G} \mathrm{GL}_{n}$ denotes the quotient of $U \times \mathrm{GL}_{n}$ by $G$, acting as given on $U$ and by left translation on $\mathrm{GL}_{n}$, which is an algebraic space. So, the definition of quotient stack is unchanged if we restrict to $\mathrm{GL}_{n}$-actions. According to [7, Lemma 2.12], a quotient stack can be characterized by the existence of a vector bundle with faithful actions of the geometric stabilizer group schemes. Returning to (2.1), we remark that the projection from $U \times{ }^{G} \mathrm{GL}_{n}$ to $G \backslash \mathrm{GL}_{n}$ is étale locally the projection from a product with $U$. When $G$ is reductive, then, $U$ affine implies $U \times{ }^{G} \mathrm{GL}_{n}$ affine.

\subsection{Boundary Components}

Let $\overline{\mathcal{C}}_{g} \rightarrow \overline{\mathcal{M}}_{g}$ be the universal family over the moduli stack of Deligne-Mumford curves of genus $g$. The algebraic stacks $\overline{\mathcal{C}}_{g}$ and $\overline{\mathcal{M}}_{g}$ are smooth over $\operatorname{Spec}(k)$. We 
will denote the relative singular locus by

$$
D_{g}:=\overline{\mathcal{C}}_{g}^{\text {sing }},
$$

which is defined by the Fitting ideal of the sheaf of relative differentials. The algebraic stack $D_{g}$ is smooth of codimension 2 in $\overline{\mathcal{C}}_{g}$ (cf. $[6, \S 1]$ ) and is the normalization of the boundary divisor of $\overline{\mathcal{M}}_{g}$.

The irreducible components of the boundary divisor of $\overline{\mathcal{M}}_{g}$ are indexed by unordered pairs of positive integers summing to $g$ and an additional element (labeled "irr" in [4]).

There is a degree 2 étale cover $\widehat{D}_{g} \rightarrow D_{g}$ where $\widehat{D}_{g}$ parameterizes stable curves together with a node and a choice of tangent direction to the curve at the node. We recall from [4, XII.10.11] that

$\widehat{D}_{g}=\overline{\mathcal{M}}_{g-1,2} \sqcup\left(\overline{\mathcal{M}}_{1,1} \times \overline{\mathcal{M}}_{g-1,1}\right) \sqcup\left(\overline{\mathcal{M}}_{2,1} \times \overline{\mathcal{M}}_{g-2,1}\right) \sqcup \cdots \sqcup\left(\overline{\mathcal{M}}_{g-1,1} \times \overline{\mathcal{M}}_{1,1}\right)$,

where the morphism to $D_{g}$ is given by gluing sections.

\subsection{Bad Properties of $\mathfrak{M}_{g}^{\mathrm{ss}}$}

We start by exhibiting a few exotic properties of $\mathfrak{M}_{g}^{\text {ss }}$, which indicate that $\mathfrak{M}_{g}^{\text {ss }}$ is a particularly interesting candidate to test the validity of Conjecture 1.1. In fact, we restrict our attention to the finite-type open substack $\mathfrak{M}_{g}^{\mathrm{qs}}$ of quasi-stable curves.

The inclusion $i: \overline{\mathcal{M}}_{g} \hookrightarrow \mathfrak{M}_{g}^{\mathrm{qs}}$ has complement of codimension 2 , and it follows that pullback and pushforward by stabilization $\mathrm{st}^{*}$ and $\mathrm{st}_{*}$ give an equivalence of categories of vector bundles. Since every vector bundle on $\mathfrak{M}_{g}^{\mathrm{qs}}$ is the pullback of a vector bundle on $\overline{\mathcal{M}}_{g}$, there is no vector bundle on $\mathfrak{M}_{g}^{\mathrm{qs}}$ with faithful action on the fiber by the stabilizer at a strictly semistable curve. So, by the characterization of global quotient stacks in terms of vector bundles recalled before, $\mathfrak{M}_{g}^{\mathrm{qs}}$ is not a global quotient stack. In fact, the argument of [11, Prop. 5.2] may be adapted to establish the stronger statement that $\mathfrak{M}_{g}^{\mathrm{qs}}$ does not have quasi-affine diagonal.

Now suppose that $g \geq 3$ and consider the fiber $F$ of st: $\mathfrak{M}_{g}^{\mathrm{qs}} \rightarrow \overline{\mathcal{M}}_{g}$ over a curve $C^{\prime}$ with a single node and trivial automorphism group. The fiber $F$ consists of two curves, $C^{\prime}$ and the strictly semistable curve $C$ obtained by inserting a $\mathbb{P}^{1}$ at the node. Let $U$ be a nodal cubic curve in $\mathbb{P}^{2}$. We now argue that the fiber $F$ may be identified with $\left[U / \mathbb{G}_{\mathbf{m}}\right]$. Let $\left(\widetilde{C}^{\prime}, p_{1}, p_{2}\right)$ be the pointed normalization of $C^{\prime}$ and consider the trivial family $\mathcal{X}=\widetilde{C}^{\prime} \times \mathbb{P}^{1} \rightarrow \mathbb{P}^{1}$ with sections $s_{1}, s_{2}$ corresponding to $p_{1}, p_{2}$. Here $\mathbb{G}_{m}$ acts on $\mathbb{P}^{1}$ (and on $\mathcal{X}$ ) in the standard way. Let $\widetilde{\mathcal{X}}$ be the equivariant blowup of $\mathcal{X}$ at both $p_{1}$ in the fiber over 0 and $p_{2}$ in the fiber over $\infty$, and let $\tilde{s}_{1}, \widetilde{s}_{2}$ be the proper transforms of the sections $s_{1}, s_{2}$. Now glue the sections $\widetilde{s}_{1}, \widetilde{s}_{2}$ to construct a family $\widetilde{C} \rightarrow \mathbb{P}^{1}$ such that the fibers $\widetilde{C}_{0}$ and $\widetilde{C}_{\infty}$ are isomorphic to $C$ but the generic fiber is $C^{\prime}$. Finally, since the fibers $\widetilde{C}_{0}$ and $\widetilde{C}_{\infty}$ are equivariantly isomorphic with respect to opposite actions of $\mathbb{G}_{m}$, we may glue these two fibers to construct a $\mathbb{G}_{m}$-equivariant family of curves $\mathcal{D}$ over the nodal cubic $U$. We check that the induced map from $\left[\mathcal{D} / \mathbb{G}_{m}\right]$ to the fiber $F$ of st: $\mathfrak{M}_{g}^{\mathrm{qs}} \rightarrow \overline{\mathcal{M}}_{g}$ over $C^{\prime}$ is an isomorphism (it is representable, is a 
monomorphism, and satisfies the valuative criterion for properness). It follows that there is no open substack $\mathcal{V}$ of $\mathfrak{M}_{g}^{\mathrm{qs}}$ containing $[C]$ and admitting a good moduli space.

\subsection{First Reduction}

We show that in order to establish Theorem 1.2, it suffices to show that $\mathfrak{M}_{g}^{\text {ss }}$ satisfies Conjecture 1.1.

Proposition 2.1. If $\mathfrak{M}_{g}^{\mathrm{ss}}$ satisfies Conjecture 1.1 for all $g \geq 0$, then $\mathfrak{M}_{g, n}$ satisfies Conjecture 1.1 for all $g, n \geq 0$.

Proof. An $n$-pointed prestable genus $g$ curve $\left(C,\left\{p_{i}\right\}_{i=1}^{n}\right)$ with $g$ or $n$ positive or $C$ singular is semistable (i.e., $\omega_{C}\left(\sum_{i} p_{i}\right)$ has nonnegative multidegree) if the automorphism group $\operatorname{Aut}\left(C,\left\{p_{i}\right\}_{i=1}^{n}\right)$ is linearly reductive. Therefore, to establish that $\mathfrak{M}_{g, n}$ satisfies Conjecture 1.1, it suffices to show that the moduli stack $\mathfrak{M}_{g, n}^{\text {ss }}$ of pointed semistable curves satisfies Conjecture 1.1.

For $i=1, \ldots, n$, let $C_{1}, \ldots, C_{n}$ be automorphism-free smooth 1-pointed curves of distinct genera $g_{1}, \ldots, g_{n}$ greater than $g$. Let $g^{\prime}=g+g_{1}+\cdots+g_{n}$. The morphism

$$
\mathfrak{M}_{g, n}^{\mathrm{ss}} \rightarrow \mathfrak{M}_{g^{\prime}}^{\mathrm{ss}}
$$

defined by attaching $C_{i}$ to the $i$ th marked point, is a closed immersion. If $\mathfrak{M}_{g^{\prime}}^{\text {ss }}$ satisfies Conjecture 1.1, then so does $\mathfrak{M}_{g, n}^{\mathrm{ss}}$.

\section{Quotient Structure of Algebraic Stacks}

\subsection{Stabilizer Preserving Morphisms}

A morphism $f: X \rightarrow Y$ of algebraic stacks is stabilizer preserving at a given geometric point of $X$ if it induces an isomorphism of stabilizer group schemes at that geometric point. We say that $f: X \rightarrow Y$ is pointwise stabilizer preserving if it is stabilizer preserving at all geometric points.

Lemma 3.1. Let $f: X \rightarrow Y$ be an étale representable morphism of algebraic stacks. If the fiber of $f$ over every geometric point consists of a single point, then $f$ is an isomorphism.

Proof. Since $f$ is étale and surjective, to show that $f$ is an isomorphism, it suffices to show that projection $X \times_{Y} X \rightarrow X$ is an isomorphism. The relative diagonal $X \rightarrow X \times_{Y} X$ is an isomorphism (as it is a surjective open immersion), and therefore so is $X \times_{Y} X \rightarrow X$ since the composition $X \rightarrow X \times_{Y} X \rightarrow X$ is the identity map.

LEMMA 3.2. Let $W$ and $X$ be algebraic stacks with finite stabilizer groups at geometric points, and let $f: W \rightarrow X$ be a separated morphism. If $X$ has finite inertia, then so does $W$. 
Proof. The inertia $I_{W} \rightarrow W$ factors through $I_{X} \times_{X} W$, with morphism $I_{W} \rightarrow$ $I_{X} \times_{X} W$ obtained via base change from the relative diagonal of $f$, hence finite, and $I_{X} \times_{X} W \rightarrow W$ finite by the hypothesis on $X$.

Lemma 3.3. Let $X$ be a Deligne-Mumford stack with finite inertia, let $Y$ be an algebraic stack with separated diagonal, and let $f: X \rightarrow Y$ be a morphism. Then the largest open substack $U$ of $X$ on which the restriction of $f$ is a representable morphism enjoys the following characterization: the geometric points of $U$ are precisely those at which $f$ induces an injective homomorphism of stabilizer group schemes.

Proof. Let $V \rightarrow Y$ be a smooth atlas, where $V$ is a separated scheme. The hypotheses imply that $V \rightarrow Y$ is a separated morphism. By Lemma 3.2, $X \times_{Y} V$ has finite inertia. Similarly, letting $S=V \times_{Y} V$ (so that $S$ is isomorphic to an algebraic space, with $\left(\mathrm{pr}_{1}, \mathrm{pr}_{2}\right): S \rightrightarrows V$ a groupoid presentation of $\left.Y\right), X \times_{Y} S$ has finite inertia. For a Deligne-Mumford stack with finite inertia, the largest representable open substack is the complement of the image of the complement of the identity in the inertia stack, and its geometric points are characterized as those having trivial stabilizer group. The largest representable open substack of $X \times{ }_{Y} V$ has the same preimage by the maps $\operatorname{id}_{X} \times \operatorname{pr}_{i}: X \times_{Y} S \rightarrow X \times_{Y} V$ for $i=1,2$, namely the largest representable open substack of $X \times_{Y} S$, and hence determines an open substack $U$ of $X$. It is easily verified that $U$ is the largest open substack of $X$ on which the restriction of $f$ is representable and that the geometric points of $U$ are precisely those at which $f$ induces an injective homomorphism of stabilizer group schemes.

\subsection{Local Structure of Deligne-Mumford Stacks}

The following result shows that Conjecture 1.1 holds for any Deligne-Mumford stack.

Lemma 3.4. Given a Deligne-Mumford stack $X$ with separated diagonal and a point $x$ of $X$ having finite stabilizer:

(i) There exist an affine scheme $W$, finite group $G$, action of $G$ on $W$, étale representable morphism $f:[W / G] \rightarrow X$, and point $y \in[W / G]$ such that $f(y)=x$ and $f$ is stabilizer preserving at $y$.

(ii) The group $G$ in (i) may be taken to be the geometric stabilizer group at $x$.

Proof. We obtain (i) from [18, Prop. 6.11] and [12, Thm. 6.1]. For (ii), a variation of the argument of [18, Prop. 6.11] in which $\mathscr{W}_{d}(d \in \mathbb{N})$ is replaced by $\mathscr{W}_{G}(G$ a finite group), defined as the stack over $X$ of subschemes of the pullbacks of the given étale atlas $U$, equipped with a structure of $G$-torsor, yields $V_{G} \rightarrow \mathscr{W}_{G}$ with $\mathscr{W}_{G} \cong\left[V_{G} / G\right]$. 
Remark 3.5. By [2, Prop. 3.6 and Thm. 2.19], Conjecture 1.1 holds for an algebraic stack with finite inertia. By applying $[10, \S 4]$ we see that Conjecture 1.1 in fact holds for any algebraic stack with quasi-finite and separated diagonal.

\subsection{Local Structure of Quotient Stacks}

Let $k$ be an algebraically closed field. Given a quotient stack $[U / G]$, with $U$ affine of finite type over $k$ and point $x \in U(k)$ with linearly reductive stabilizer group scheme $G_{x}$, Luna's étale slice theorem [13] gives rise (under suitable hypotheses) to an étale morphism $\left[W / G_{x}\right] \rightarrow[U / G]$, where $W$ is a $G_{x}$-invariant locally closed affine subscheme of $U$ containing $x$.

LEMMA 3.6. Let $U$ be an affine scheme of finite type over an algebraically closed field $k$, with action of a smooth linear algebraic group $G$. Then, for every point $x \in U(k)$ with linearly reductive stabilizer group scheme $G_{x}$, there exists a $G_{x}$ invariant locally closed affine subscheme $W \subset U$ containing $x$ such that the induced morphism $\left[W / G_{x}\right] \rightarrow[U / G]$ is étale. In particular, Conjecture 1.1 holds for $[U / G]$.

Proof. There exists a finite-dimensional linear $G$-space $V$ with equivariant closed immersion $U \hookrightarrow V$; thus it suffices to consider the case $U=V$. By [13, Lemma, p. 96], there exists a $G_{x}$-equivariant morphism

$$
g: V \rightarrow T_{x} V
$$

that is étale at $x$ and satisfies $g(x)=0$. We write

$$
T_{x} V=T_{x}(G \cdot x) \oplus N
$$

for a $G_{x}$-representation $N$. Then the representable morphism $\left[g^{-1}(N) / G_{x}\right] \rightarrow$ $[V / G]$ is étale at $x$ and hence on $\left[W_{0} / G_{x}\right]$ for some $G_{x}$-invariant open $W_{0} \subset$ $g^{-1}(N)$ containing $x$. Now $W$ may be taken to be any $G_{x}$-invariant affine neighborhood of $x$ in $W_{0}$.

REMARK 3.7. The conclusion of Lemma 3.6 is also valid if the hypothesis that $U$ is affine is replaced by the hypothesis that $U$ is normal. Let $x \in U(k)$ have linearly reductive stabilizer group scheme $G_{x}$. Letting $G^{\circ} \subset G$ denote the connected component of the identity, there exists a separated $G^{\circ}$-invariant open neighborhood of $x$ (e.g., the image under the action of $G^{\circ} \times U_{0}$ where $U_{0}$ is any affine open neighborhood of $x$ ). By Sumihiro's theorem [20, Thm. 3.8], there exists a quasi-projective $G^{\circ}$-invariant neighborhood $U^{\prime}$ of $x$. Letting $H$ denote the image of $G_{x}$ in $G / G^{\circ}$ and $G^{\prime}$ the preimage of $H$ under $G \rightarrow G / G^{\circ}$, if we take $h_{1}, \ldots, h_{n} \in G_{x}$ to be elements mapping onto $H$, then $h_{1} U^{\prime} \cap \cdots \cap h_{n} U^{\prime}$ is a $G^{\prime}$-invariant quasi-projective neighborhood of $x$. So we are reduced to the case that $U$ is normal and quasi-projective. Then there is an equivariant immersion $U \hookrightarrow \mathbb{P}(V)$ for some finite-dimensional linear $G$-space $V$, and as in the proof of Lemma 3.6, we are further reduced to the case $U=\mathbb{P}(V)$. We conclude by taking 
$f$ to be a $G_{x}$-semi-invariant homogeneous polynomial with $f(x) \neq 0$, invoking [13, Lemma, p. 96] to obtain $G_{x}$-equivariant

$$
g: \mathbb{P}(V)_{f} \rightarrow T_{x} \mathbb{P}(V),
$$

étale at $x$ with $g(x)=0$, and applying the rest of the proof of Lemma 3.6.

LEMMA 3.8. Let $Y$ be an algebraic stack locally of finite type over an algebraically closed field $k$. If there is a finite, flat cover $f: X=\left[U / \mathrm{GL}_{n}\right] \rightarrow Y$ for some $n$, where $U$ is an algebraic space (resp., an affine scheme), then $Y \cong\left[V / \mathrm{GL}_{n^{\prime}}\right]$ for some $n^{\prime}$, where $V$ is an algebraic space (resp., an affine scheme).

Proof. If $E$ is a vector bundle on $X$ such that the stabilizer at every geometric point acts faithfully on the fiber, then $f_{*} E$ is a vector bundle on $Y$ with the same property (cf. [7, proof of Lemma 2.13]). If $U$ is affine, the base change $X \times_{Y} V$ is also an affine scheme-indeed, since $U \rightarrow X$ is a $\mathrm{GL}_{n}$-bundle and $X \times_{Y} V \rightarrow X$ is a $\mathrm{GL}_{n^{\prime}}$-bundle, the base change $U \times_{Y} V \rightarrow X \times_{Y} V$ is a $\mathrm{GL}_{n}$-bundle, and since $U \times{ }_{Y} V$ is affine, so is $X \times_{Y} V$. Since $X \times_{Y} V \rightarrow V$ is finite and surjective, $V$ is an affine scheme.

REMARK 3.9. In $[19, \S 2]$, Rydh defines an algebraic stack $X$ of finite presentation over $k$ to be of global type if every point $x \in X(k)$ is in the image of an étale, representable morphism $\left[W / \mathrm{GL}_{n}\right] \rightarrow X$ where $W$ is quasi-affine. Any algebraic stack satisfying Conjecture 1.1 that is also quasi-compact and has linearly reductive stabilizers at closed points is therefore of global type.

\subsection{Second Reduction}

We give a result reducing Conjecture 1.1 for $\mathfrak{M}_{g}^{\text {ss }}$ to exhibiting certain finite covers of étale neighborhoods.

Proposition 3.10. Let $X$ be an algebraic stack, locally of finite type over an algebraically closed field $k$ with separated and quasi-compact diagonal such that $X$ has affine stabilizer groups at all closed points. Suppose that $x \in X(k)$ has linearly reductive stabilizer group scheme $G_{x}$, and there exist morphisms

$$
X^{\prime \prime} \rightarrow X^{\prime} \rightarrow X
$$

and $x^{\prime} \in X^{\prime}(k)$ such that

(i) $X^{\prime \prime} \cong\left[U / \mathrm{GL}_{n}\right]$ for some affine scheme $U$ over $k$ with action of $\mathrm{GL}_{n}$ for some $n$,

(ii) $X^{\prime \prime} \rightarrow X^{\prime}$ is a finite flat cover,

(iii) $X^{\prime} \rightarrow X$ is étale representable,

(iv) $X^{\prime} \rightarrow X$ is stabilizer preserving at $x^{\prime}$.

Then Conjecture 1.1 holds for $X$ at the point $x$.

Proof. By Lemma 3.8 we have $X^{\prime} \cong\left[V / G L_{n^{\prime}}\right]$. We conclude by Lemma 3.6. 


\section{Proof in a Special Case}

We apply the previous reduction steps (Propositions 2.1 and 3.10) and a structural result (Lemma 4.3) to give a proof of Theorem 1.2 in a special case. Namely, we will prove Theorem 4.2 that asserts that Conjecture 1.1 holds near semistable curves whose stabilization is the nodal union of two smooth curves of different genera. We remark that our argument in this section does not apply to semistable curves whose stabilization has precisely one node but admits an automorphism that swaps the tangent branches at the node. Therefore, we exclude curves whose stabilization is an irreducible curve with precisely one node or is the nodal union of two smooth curves of the same genus.

\subsection{Aligned log Structure}

We recall the definition of an aligned $\log$ structure introduced in [1]. Let $X$ be a scheme. Given a $\log$ structure $M \rightarrow \mathcal{O}_{X}$ we consider the characteristic sheaf $\bar{M}=M / \mathcal{O}_{X}^{*}$. If $M$ is a locally free $\log$ structure, then there is a subsheaf of sets $\bar{M}^{1} \subset \bar{M}$ whose values on stalks are the sums of subsets of generators of the free monoids $\bar{M}_{\bar{x}}$ at geometric points $\bar{x} \in X$. An aligned log structure is a locally free $\log$ structure together with a subsheaf $\mathcal{S} \subset \bar{M}^{1}$ such that, for every geometric point $\bar{x} \in X$, there is a labeling of the generators of $\bar{M}_{\bar{x}}$ as $e_{1}, \ldots, e_{n}$ such that

$$
\mathcal{S}_{\bar{x}}=\left\{0, e_{1}, e_{1}+e_{2}, \ldots, e_{1}+\cdots+e_{n}\right\} .
$$

Proposition 4.1. Let $E$ be a Deligne-Mumford stack, $E \rightarrow \overline{\mathcal{M}}_{g}$ an étale morphism with corresponding family of curves $C \rightarrow E$, and $D \subset C^{\operatorname{sing}}$ an open and closed subscheme that maps isomorphically to a divisor in E. Define

$$
X:=E \times \overline{\mathcal{M}}_{g} \mathfrak{M}_{g}^{\mathrm{ss}},
$$

and let $\mathfrak{C} \rightarrow X$ denote the associated family of semistable curves, with stabilization $\mathfrak{C} \rightarrow C \times{ }_{E} X$.

(i) The stack

$$
D \times C^{\text {sing }} \mathfrak{C}^{\text {sing }}
$$

is, by stabilization, the normalization of $D \times_{E} X$.

(ii) Suppose that the family of curves $D \times{ }_{E} C \rightarrow D$ is obtained by gluing some $C_{0} \rightarrow D$ along two sections $s_{1}$ and $s_{2}$, as in Section 2.1. Then there is a unique aligned log structure $(M, S)$ on $X$ whose underlying log structure is that of the normal crossing divisor $D \times_{E} X$ such that, for every geometric point $\bar{x}$ of $D \times_{E} X$ corresponding to a prestable curve whose stabilization collapses $r$ exceptional components to the corresponding point of $D$, the elements of $S_{\bar{x}}$ under the isomorphism of (i) are $\bar{x}_{1}+\cdots+\bar{x}_{j}$ for $0 \leq j \leq r+1$, where $\bar{x}_{1}, \ldots, \bar{x}_{r+1}$ are the points of $\mathfrak{C}^{\text {sing }}$ mapping to the point of $D$, ordered so that $\bar{x}_{1}$ is the image of $s_{1}$ under the rational map $\left(C_{0}\right)_{\bar{x}} \rightarrow-\mathfrak{C}_{\bar{x}}$, and for every $i$, the points $\bar{x}_{i}$ and $\bar{x}_{i+1}$ lie on one of the exceptional components collapsing to the point of $D$ corresponding to $\bar{x}$. 
Proof. Since $D \times{ }_{C}^{\text {sing }} C^{\text {sing }}$ is smooth and the morphism to $D \times_{E} X$ is finite and restricts to an isomorphism over the stable curves in $X$, assertion (i) is clear.

Assertion (ii) quickly reduces to the following claim. Let $R$ be a Henselian discrete valuation ring with residue field $k$, together with $C_{0, R} \rightarrow \operatorname{Spec}(R)$ with two sections and $\mathfrak{C}_{R} \rightarrow \operatorname{Spec}(R)$, corresponding to some $\operatorname{Spec}(R) \rightarrow D \times_{E} X$; in particular, the stabilization of $\mathfrak{C}_{R}$ is assumed to be identified with the stable curve $C_{R}$ obtained by gluing from $C_{0, R} \rightarrow \operatorname{Spec}(R)$ with the two sections. Denoting by $s_{1, R}$ and $s_{2, R}$ the two sections, the common composite with the gluing is a section $\operatorname{Spec}(R) \rightarrow C_{R}$ with image contained in $C_{R}^{\operatorname{sing}}$. The restriction

$$
h: \operatorname{Spec}(R) \times{ }_{C_{R}^{\text {sing }}} \mathfrak{C}_{R}^{\text {sing }} \rightarrow \operatorname{Spec}(R)
$$

of the finite unramified morphism of (i) is necessarily the projection of a disjoint union of some copies of $\operatorname{Spec}(R)$ and some (possibly nonreduced) points mapping to the closed point of $\operatorname{Spec}(R)$. Let the fiber over the closed point be $x_{1}, \ldots, x_{r+1}$ as in (ii), and let

$$
i_{1}<i_{2}<\cdots<i_{q+1}
$$

be the indices of those points over the closed point that are the specializations of copies of $\operatorname{Spec}(R)$. The claim is that at a geometric general point $\bar{\eta}$ of $\operatorname{Spec}(R)$, stabilization of $\mathfrak{C}_{\bar{\eta}}$ collapses a chain of $q$ rational curves to the node of $C_{\bar{\eta}}$ (the common image of the two sections under gluing), and with the points $\bar{\eta}_{1}, \ldots, \bar{\eta}_{q+1}$ as in (ii), the point $\bar{\eta}_{a}$ maps to the $i_{a}$-copy of $\operatorname{Spec}(R)$ for $a=1, \ldots, q+1$.

There is nothing to prove if $q=0$, so we assume that $q \geq 1$. There is an iterated blow-up $\tau: \widehat{C}_{0, R} \rightarrow C_{0, R}$ at points over $c_{1}:=s_{1, R}(\operatorname{Spec}(k))$ and $c_{2}:=$ $s_{2, R}(\operatorname{Spec}(k))$ such that the composite with gluing and the inverse of stabilization

$$
\widehat{C}_{0, R} \stackrel{\tau}{\rightarrow} C_{0, R} \rightarrow C_{R}-\rightarrow \mathfrak{C}_{R}
$$

is defined in a neighborhood of $\tau^{-1}\left(\left\{c_{1}, c_{2}\right\}\right)$. (The process of blowing up points of indeterminacy terminates. A classical result of Northcott asserts that upon blowing up and replacing an $\mathfrak{m}_{c_{i}}$-primary ideal in $\mathcal{O}_{C_{0, R}, c_{i}}$ by its proper transform the multiplicity decreases; cf. [9].) Now $\tau^{-1}\left(c_{1}\right)$ is a connected scheme whose image $Z_{0} \subset \mathfrak{C}_{R}$ contains $x_{1}$ and the specialization of $\eta_{1}$.

For each $1 \leq a \leq q$, the generic fiber $\mathfrak{C}_{\eta}$ has a unique exceptional component $E_{\eta, a}$ containing $\eta_{a}$ and $\eta_{a+1}$. We let $W_{a}$ denote the closure of $E_{\eta, a}$ in $\mathfrak{C}_{R}$. The special fiber $Z_{a}$ of $W_{a}$ is connected and contains the specializations of $\eta_{a}$ and $\eta_{a+1}$.

Since $\mathfrak{C}_{R}$ is Cohen-Macaulay with reduced special fiber, the complement of the closure of $\mathfrak{C}_{\eta}^{\text {sing }}$ is normal. In particular, each $x_{i}$ with $i \notin\left\{i_{1}, \ldots, i_{q+1}\right\}$ is a normal point of $\mathfrak{C}_{R}$, as are all smooth points of the special fiber of $\mathfrak{C}_{R}$.

With these observations, we may establish the claim by contradiction. Suppose there is some $a$, which we take to be minimal, such that $\eta_{a}$ specializes to $x_{i}$ and $\eta_{a+1}$ specializes to $x_{j}$, with $j<i$. If $a=1$, then since $Z_{0}$ is connected and contains $x_{1}$ and $x_{i}$, we have $x_{j} \in Z_{0}$ as well, and then since $Z_{1}$ is connected and one-dimensional, we would have $\operatorname{dim}\left(Z_{0} \cap Z_{1}\right)=1$, which is a contradiction. If 
$a>1$, then we may argue similarly, using that $Z_{0} \cup \cdots \cup Z_{a-1}$ is connected and contains $x_{1}$ and $x_{i}$ and hence must as well contain $x_{j}$.

\subsection{Separating Nodes with Distinct Genera}

From Section 2.1, every partition of $g$ as a sum of two positive integers determines an irreducible component of the boundary divisor of $\overline{\mathcal{M}}_{g}$. Suppose that $g_{1}+g_{2}=g$ with $g_{1}>g_{2}$. Then the corresponding boundary component has an open substack $D_{g_{1}, g_{2}}$ parameterizing unions of smooth curves of genera $g_{1}$ and $g_{2}$ at a node. We define

$$
E_{g_{1}, g_{2}}:=\mathcal{M}_{g} \cup D_{g_{1}, g_{2}} \subset \overline{\mathcal{M}}_{g},
$$

the open substack consisting of smooth curves of genus $g$ and unions of smooth curves of genera $g_{1}$ and $g_{2}$. Then the inclusion of $E_{g_{1}, g_{2}}$ in $\overline{\mathcal{M}}_{g}$ and $D_{g_{1}, g_{2}}$ satisfy the hypotheses of Proposition 4.1 and hence determine an aligned log structure on

$$
X_{g_{1}, g_{2}}:=E_{g_{1}, g_{2}} \times \overline{\mathcal{M}}_{g} \mathfrak{M}_{g}^{\text {ss }},
$$

that is, a morphism

$$
X_{g_{1}, g_{2}} \rightarrow \mathcal{L} o g^{\mathrm{al}}
$$

to the stack of aligned log structures; the stack of aligned log structures is described in [1].

Theorem 4.2. For positive integers $g_{1}, g_{2}$, and $g$ with $g_{1}+g_{2}=g$ and $g_{1}>g_{2}$, Conjecture 1.1 holds for $X_{g_{1}, g_{2}}$.

The key to the proof is the following observation, which reveals the structure of the moduli stack of semistable curves of genus $g$ near a curve whose stabilization is a union of smooth curves of genera $g_{1}$ and $g_{2}$. The quotient stack

$$
\mathcal{A}^{1}:=\left[\mathbb{A}^{1} / \mathbb{G}_{m}\right],
$$

where $\mathbb{G}_{m}$ acts in the standard way on $\mathbb{A}^{1}$, plays an important role. As is recalled in [1], the stack $\mathcal{A}^{1}$ serves as a universal target for pairs consisting of a smooth variety or algebraic stack $Y$ with divisor $D \subset Y$. If $D$ is also smooth, then the corresponding morphism $Y \rightarrow \mathcal{A}^{1}$ is smooth.

LEMмA 4.3. The projection from $X_{g_{1}, g_{2}}$ and morphism (4.1) fit into a fiber square

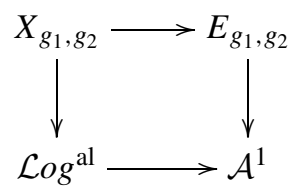

where the morphisms to $\mathcal{A}^{1}$ correspond to the boundary divisor of $\mathcal{L}^{\mathrm{al}}{ }^{\mathrm{al}}$ and the divisor $D_{g_{1}, g_{2}} \subset E_{g_{1}, g_{2}}$. 
Proof. The horizontal morphisms restrict to isomorphisms on the complements of substacks of codimension 2, hence so does the morphism

$$
X_{g_{1}, g_{2}} \rightarrow \mathcal{L}_{o g}^{\mathrm{al}} \times \mathcal{A}^{1} E_{g_{1}, g_{2}},
$$

which is representable. The fiber product in (4.2) is smooth since the morphism $E_{g_{1}, g_{2}} \rightarrow\left[\mathbb{A}^{1} / \mathbb{G}_{m}\right]$ is smooth. So, by Zariski-Nagata purity [8, Thm. X.3.1] the morphism (4.2) is étale. The morphism (4.2) is bijective on geometric points and pointwise stabilizer preserving and hence by Lemma 3.1 is an isomorphism.

For the proof of Theorem 4.2, we use the stacks

$$
\mathcal{A}^{n}:=\left[\mathbb{A}^{n} / \mathbb{G}_{m}^{n}\right],
$$

also introduced in [1] (quotient stacks for the standard group actions).

Proof of Theorem 4.2. Let $x \in X_{g_{1}, g_{2}}(k)$ correspond to a semistable curve $C$ of genus $g$ whose stabilization $C^{\prime}$ is the union of smooth curves of genera $g_{1}$ and $g_{2}$. We verify Conjecture 1.1 for $X_{g_{1}, g_{2}}$ at $x$. Let $H$ be the automorphism group of $C^{\prime}$. By Lemma 3.4 there exist an affine scheme $W$ with action of $H$ fixing a point $y \in W$ and an étale representable morphism

$$
[W / H] \rightarrow E_{g_{1}, g_{2}}
$$

sending $y$ to the point of $E_{g_{1}, g_{2}}$ corresponding to $C^{\prime}$.

If $C$ is stable, then we are done, so we assume that some positive number $r$ of exceptional components are collapsed by stabilization $C^{\prime} \rightarrow C$. The coordinate hyperplanes give rise to an aligned $\log$ structure on $\mathcal{A}^{r+1}$, and the corresponding morphism $\mathcal{A}^{r+1} \rightarrow \mathcal{L} g^{\text {al }}$ is étale representable [1] and stabilizer preserving at the origin. Consequently, we have a stack

$$
X^{\prime}:=\mathcal{A}^{r+1} \times{ }_{\mathcal{L} o g} X_{g_{1}, g_{2}} \times_{E_{g_{1}, g_{2}}}[W / H],
$$

such that the projection to $X_{g_{1}, g_{2}}$ is étale representable, and a point $x^{\prime} \in X^{\prime}$ at which the projection to $X_{g_{1}, g_{2}}$ is stabilizer preserving, sending $x^{\prime}$ to $x$.

We let

$$
X^{\prime \prime}:=\mathcal{A}^{r+1} \times \mathcal{L}_{\text {og }^{\mathrm{al}}} X_{g_{1}, g_{2}} \times_{E_{g_{1}, g_{2}}} W .
$$

By Lemma $4.3, X^{\prime \prime} \cong \mathcal{A}^{r+1} \times \mathcal{A}^{1} W$. Since $W \rightarrow \mathcal{A}^{1}$ is affine, $X^{\prime \prime}$ is of the form $\left[U / \mathbb{G}_{m}^{r+1}\right]$ with $U$ affine, and Proposition 3.10 implies Conjecture 1.1 for $X_{g_{1}, g_{2}}$ at $x$.

\section{Stacks Generalities}

In this section, we record general facts about algebraic stacks that will be useful for the proof of Theorem 1.2. The reader may want to skip this section on the first reading but later return when these results are applied in Sections 6 through 8 . 


\section{1. Étale Coverings from Unramified Morphisms}

Given a representable unramified morphism $X \rightarrow Y$ of algebraic stacks, Rydh constructs [17, §3] a stack $E_{X / Y}$ and a factorization $X \rightarrow E_{X / Y} \rightarrow Y$ as a closed immersion followed by an étale representable morphism. ${ }^{1}$ The complement of $X$ in $E_{X / Y}$ is isomorphic to $Y$. The construction, known as the étale envelope, is functorial: given a scheme $T$, a morphism $T \rightarrow E_{X / Y}$ consists of a morphism $T \rightarrow Y$ with closed subscheme $T^{\prime} \subset T$ and open immersion $T^{\prime} \rightarrow X \times_{Y} T$ over $T$. For example, if $X \rightarrow Y$ is étale, then (see [17, Exa. 2.1]) $E_{X / Y}$ is the disjoint union of $X$ and $Y$. If $X$ is the disjoint union of a pair of distinct lines $\ell_{1}$, $\ell_{2}$ in the plane $Y=\mathbb{P}^{2}$, then (see [17, Exa. 2.2]) $E_{X / Y}$ is the scheme covered by three copies $Y_{0}, Y_{1}, Y_{2}$ of $Y$ with $Y_{0}$ and $Y_{i}$ glued along the complement of $\ell_{i}$ for $i=1,2$; notice that the fiber of $E_{X / Y} \rightarrow Y$ over $\ell_{1} \cap \ell_{2}$ consists of three points, all contained in the closure of $\ell_{1} \cup \ell_{2} \subset Y=Y_{0}$.

We need, as well, a variant due to Mustaţă and Mustaţă $[14, \S 1.1]$. Suppose that $X \rightarrow Y$ is a representable unramified morphism of algebraic stacks that is étale onto its image, a locally closed substack $Y_{1} \subset Y$. The topological closure $\bar{Y}_{1}$ is then closed in $E_{X / Y}$, as we see by combining the isomorphism $E_{X^{\text {red }} / Y} \cong E_{X / Y}$ of [17, Thm. 1.2(ix)] with the consequence of functoriality that $E_{X^{\text {red }} / Y_{1}^{\text {red }}} \rightarrow E_{X^{\text {red }} / Y}$ is a closed immersion. Then we define

$$
F_{X / Y}:=E_{X / Y} \backslash \bar{Y}_{1} .
$$

From the properties of the étale envelope mentioned before we obtain, for $F_{X / Y}$, a factorization $X \rightarrow F_{X / Y} \rightarrow Y$ as a closed immersion followed by an étale representable morphism and a functorial description, where for a scheme $T$, a morphism $T \rightarrow F_{X / Y}$ is given by a morphism $T \rightarrow Y$ whose image is disjoint from $\bar{Y}_{1} \backslash Y_{1}$ and an open immersion $Y_{1} \times_{Y} T \rightarrow X \times_{Y} T$ over $T$.

Proposition 5.1. Let $f: X \rightarrow Y$ be an étale representable morphism of algebraic stacks. For a locally closed substack $W \subset Y$ with $f(X) \cap \bar{W} \subset W$ and fiber diagram

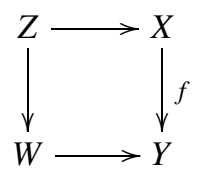

the induced morphism $g: X \rightarrow F_{Z / Y}$ is an isomorphism if and only if the restriction of $f$ over $Y \backslash \bar{W}$ is an isomorphism.

Proof. The forward implication is clear from the definition of $F_{Z / Y}$. The reverse implication follows by applying Lemma 3.1 to $g$ by noting that the geometric points of both $X$ and $F_{Z / Y}$ lie over $Y \backslash(\bar{W} \backslash W)$ and observing that $g$ restricts to isomorphisms over $W$ and over $Y \backslash \bar{W}$.

\footnotetext{
${ }^{1}$ Rydh works in greater generality, not requiring $X \rightarrow Y$ to be representable.
} 


\subsection{Some Stacks with Nonseparated Diagonal}

The stack of log structures [15] has quasi-compact but nonseparated diagonal. Here we describe an algebraic stack $\stackrel{\mathcal{A}}{r}^{r}$ for each $r>0$, also with quasi-compact but nonseparated diagonal.

We recall, from Section 4.2, the stack $\mathcal{A}^{r}=\left[\mathbb{A}^{r} / \mathbb{G}_{m}^{r}\right]$ (quotient stack for the standard action of $\mathbb{G}_{m}^{r}$ on $\mathbb{A}^{r}$ ). There are an involution $\iota: \mathbb{A}^{r} \rightarrow \mathbb{A}^{r}$, $\left(x_{1}, \ldots, x_{r}\right) \mapsto\left(x_{r}, \ldots, x_{1}\right)$ and a similar compatible involution of $\mathbb{G}_{m}^{r}$ inducing an involution $\iota: \mathcal{A}^{r} \rightarrow \mathcal{A}^{r}$.

Let us introduce $\mathcal{A}^{r}$ by defining $U_{r}$ to be the nonseparated scheme that is the union of two copies of $\mathbb{A}^{r} \times \mathbb{G}_{m}^{r}$ along the two copies of $\left(\mathbb{A}^{1} \backslash\{0\}\right)^{r} \times \mathbb{G}_{m}^{r}$ identified via the involution

$$
\left(x_{1}, \ldots, x_{r}, t_{1}, \ldots, t_{r}\right) \mapsto\left(x_{1}, \ldots, x_{r}, t_{r} \frac{x_{r}}{x_{1}}, \ldots, t_{1} \frac{x_{1}}{x_{r}}\right)
$$

and setting

$$
\stackrel{\circ}{\mathcal{A}}^{r}=\left[U_{r} \rightrightarrows \mathbb{A}^{r}\right],
$$

the stack associated to the groupoid scheme with projection map and twisted action map, which on the first copy of $\mathbb{A}^{r} \times \mathbb{G}_{m}^{r}$ is the standard diagonal action and on the second copy is the composition of the involution $\iota$ of $\mathbb{A}^{r}$ with the standard diagonal action.

The stabilizer of $\stackrel{\circ}{\mathcal{A}}^{r}$ at the origin is the semidirect product $\mathbb{G}_{\mathbf{m}}^{r} \rtimes \mathbb{Z} / 2 \mathbb{Z}$ where $\mathbb{Z} / 2 \mathbb{Z}$ acts on $\mathbb{G}_{\mathbf{m}}^{r}$ via the involution $\left(t_{1}, \ldots, t_{r}\right) \mapsto\left(t_{r}, \ldots, t_{1}\right)$ of $\mathbb{G}_{\mathbf{m}}^{r}$.

Just as the stack $\mathcal{A}^{1}$ has the well-known interpretation of schemes with a line bundle and a global section, we leave it to the reader to see that $\stackrel{\mathcal{A}}{1}^{1}$ has the interpretation of schemes with a line bundle, a global section, and a degree-two finite étale cover of the zero-locus of the section. In particular, the fiber of the obvious morphism $\stackrel{\circ}{A}^{1} \rightarrow \mathcal{A}^{1}$ over $B \mathbb{G}_{m}=\left[\{0\} / \mathbb{G}_{m}\right]$ is isomorphic to $B \mathbb{G}_{m} \times B \mathbb{Z} / 2 \mathbb{Z}$; Proposition 5.1 yields an isomorphism

$$
F_{B \mathbb{G}_{m} / \stackrel{\mathcal{A}}{1}^{1}} \cong \mathcal{A}^{1}
$$

Multiplication of coordinates induces morphisms mul: $\mathcal{A}^{r} \rightarrow \mathcal{A}^{1}$ and mul: $\stackrel{\circ}{\mathcal{A}}^{r} \rightarrow \stackrel{\circ}{\mathcal{A}}^{1}$. There are étale representable morphisms $\mathcal{A}^{r} \rightarrow \stackrel{\circ}{\mathcal{A}}^{r}$ induced on the level of groupoid schemes from the inclusion of the first copy of $\mathbb{A}^{r} \times \mathbb{G}_{m}^{r}$ in $U_{r}$.

Proposition 5.2. These morphisms fit into a fiber diagram

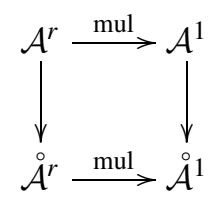

Proof. Each of the morphisms arises from a morphism of groupoid schemes. Standard manipulations of groupoid schemes establish the proposition. 


\subsection{Semi-aligned log Structures}

We return to the setting of Section 4.1. Besides the zero section, there is another global section $s_{1}$ of $\bar{M}^{1}$, given by the sums of generators of the $\bar{M}_{\bar{x}}$. An involution $j$ of $\bar{M}^{1}$ is characterized by the property that the composite

$$
\bar{M}^{1} \stackrel{(\mathrm{id}, j)}{\longrightarrow} \bar{M}^{1} \times \bar{M}^{1} \subset \bar{M} \times \bar{M} \stackrel{+}{\longrightarrow} \bar{M}
$$

is the constant map $s_{1}$. Given an aligned log structure, application of the involution $j$ yields a new aligned log structure, the opposite aligned log structure.

A related notion is semi-aligned log structure, exactly as in Section 4.1, but with

$$
\mathcal{S}_{\bar{x}}=\left\{0, e_{1}, e_{n}, e_{1}+e_{2}, e_{n-1}+e_{n}, \ldots, e_{1}+\cdots+e_{n}\right\} .
$$

Proposition 5.3. Let $\pi: X \rightarrow Y$ be a finite type étale universally closed morphism of algebraic spaces. Then there is a natural transformation

$$
\operatorname{tr}: \pi_{*} \circ \pi^{*} \rightarrow \mathrm{id}
$$

("trace") on sheaves of idempotent monoids on $Y$, which on stalks is given by summing the values on fibers.

Proof. Given $y \in Y$, there exist an étale neighborhood $y^{\prime} \in Y^{\prime} \rightarrow Y$ with $y^{\prime} \mapsto$ $y$ and sections $\sigma_{1}, \ldots, \sigma_{r}$ of $\pi^{\prime}: X^{\prime}:=X \times_{Y} Y^{\prime} \rightarrow Y^{\prime}$ whose images cover $\pi^{\prime-1}\left(y^{\prime}\right)$. Since $\pi$ is universally closed, we may replace $Y^{\prime}$ by a suitable open neighborhood of $y^{\prime}$, so that the images of the sections cover $X^{\prime}$. We may then define the trace locally by summing the $r$ sections over $Y^{\prime}$.

Besides the stack of aligned log structures $\log ^{\text {al }}$ described in [1], there is a stack of semi-aligned $\log$ structures, which we denote by $\log ^{(1 / 2) a l}$. The assignment, to a scheme with an aligned log structure, of the opposite aligned $\log$ structure, yields an involution $j$ of the stack $\mathcal{L o g}^{\mathrm{al}}$. If $X$ is a scheme and $\left(M \rightarrow \mathcal{O}_{X}, \mathcal{S} \subset \bar{M}^{1}\right)$ is an aligned $\log$ structure on $X$, then $\mathcal{S} \sqcup j(\mathcal{S})$ is an aligned $\log$ structure on $X \sqcup X$, and the trace for $X \sqcup X \rightarrow X$ yields $\operatorname{tr}(\mathcal{S} \sqcup j(\mathcal{S})$ ), a semialigned $\log$ structure on $X$. This construction gives rise to a morphism of algebraic stacks

$$
\mathcal{L}_{o g}{ }^{\mathrm{al}} \rightarrow \mathcal{L}^{(1 / 2) \mathrm{al}} .
$$

Proposition 5.4. The morphism (5.2) is representable, étale, of finite type, and universally closed, and restricts to an isomorphism over the locus of locally free $\log$ structures of rank $\leq 1$ and a finite étale morphism of degree 2 over the locus of rank $\geq 2$.

Proof. The morphism (5.2) is clearly representable and locally of finite type, and it is trivial to verify the criterion in terms of square-zero extensions to be étale. The sheaf of sections of (5.2) is locally the quotient of a finite constant sheaf; hence, the morphism (5.2) is universally closed of finite type. The assertion about the locus of locally free $\log$ structures of rank $\leq 1$ is a triviality. Since the geometric 
fibers over locally free $\log$ structures of rank $\geq 2$ all consist of two points, the properties of the morphism (5.2) imply that it restricts to a finite morphism.

Given a normal crossings divisor $D$ on a smooth algebraic stack $X$, we set $X^{k} \subset X$ to be the locally closed substack consisting of all points lying, smooth locally in exactly $k$ smooth divisors. For instance, $X^{0}=X \backslash D$, and $X^{1}$ is the smooth locus of $D$. Set $X^{\leq k}$ and $X^{\geq k}$ to be the locally closed substacks obtained by taking the unions of the corresponding $X^{i}$. We make these definitions more generally for an algebraic stack with a given locally free log structure.

Considering the fiber square

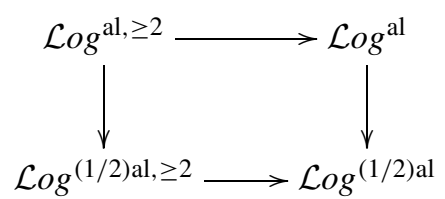

Proposition 5.4 tells us that the criterion of Proposition 5.1 is satisfied.

COROLlaRY 5.5. The fiber square (5.3) induces an isomorphism

$$
\mathcal{L}_{o g}{ }^{\mathrm{al}} \rightarrow F_{\mathcal{L} g^{\mathrm{al}, \geq 2} / \mathcal{L}_{\log }(1 / 2) \mathrm{al}} .
$$

Another consequence of Proposition 5.4 is that we have a morphism

$$
\text { al: } \log ^{(1 / 2) \mathrm{al}, \geq 2} \rightarrow B \mathbb{Z} / 2 \mathbb{Z}
$$

corresponding to the restriction of morphism (5.2) to $\log ^{(1 / 2) a 1, \geq 2}$. Now we consider the composite morphism

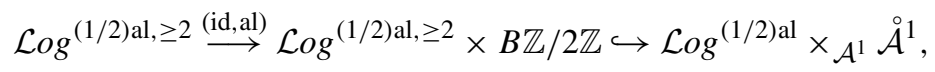

remembering that the fiber of $\mathcal{A}^{1}$ over $B \mathbb{G}_{m} \subset \mathcal{A}^{1}$ is isomorphic to $B \mathbb{G}_{m} \times$ $B \mathbb{Z} / 2 \mathbb{Z}$, so that we obtain a stack

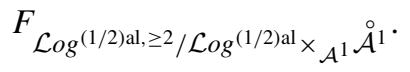

We note that whereas $\mathcal{L}_{\log }{ }^{\mathrm{al}, r}$ consists of a single point with stabilizer $\mathbb{G}_{\mathbf{m}}^{r}$ and $\mathcal{L} \operatorname{sog}^{(1 / 2) a l, r}$ consists of a single point with stabilizer $\mathbb{G}_{\mathbf{m}}^{r}$ for $r \leq 1$ and $\mathbb{G}_{\mathbf{m}}^{r} \rtimes \mathbb{Z} / 2 \mathbb{Z}$ for $r \geq 2$, the stack (5.5) in codimension $r$ is a point with trivial stabilizer for $r=0$ and stabilizer $\mathbb{G}_{\mathbf{m}}^{r} \rtimes \mathbb{Z} / 2 \mathbb{Z}$ for $r \geq 1$. This feature leads us to introduce a new notion and compact notation.

By an augmented semi-aligned log structure on a scheme or algebraic stack $X$ we mean the data of a semi-aligned $\log$ structure $(M, S)$, a degree 2 finite étale cover $\widehat{X} \rightarrow X^{\geq 1}$, and an augmentation isomorphism

$$
\widehat{X}^{\geq 2} \rightarrow \mathcal{L}^{\mathrm{al}} \times_{\mathcal{L}^{(1 / 2) \text { al }} X^{\geq 2}}
$$

over $X^{\geq 2}$. (The fiber product is of the morphism (5.2) and the restriction of $(M, S)$ to $X^{\geq 2}$.) The stack (5.5) is identified with the stack of augmented semi-aligned 
$\log$ structures, which we denote by

$$
\log ^{(1 / 2+\varepsilon) \mathrm{al}} \text {. }
$$

To understand the stack (5.5), following the definition in Section 5.1, it is important to remember the isomorphism of target objects in the definition of fiber product of stacks $\log ^{(1 / 2) a l, \geq 2} \times{ }_{\mathcal{L}_{o g}(1 / 2) a l} \times_{\mathcal{A}^{1}} \stackrel{\circ}{\mathcal{A}}^{1} T$ (target of the open immersion).

As an example, there is a natural semi-aligned log structure on $\AA^{r}$, by taking the $e_{1}, \ldots, e_{n}$ to correspond to the natural coordinates on $\mathbb{A}^{r}$. The triple consisting of this semi-aligned $\log$ structure, the cover $\left(\mathcal{A}^{r}\right)^{\geq 1} \rightarrow\left(\stackrel{\circ}{\mathcal{A}}^{r}\right)^{\geq 1}$, and the restriction of the natural aligned $\log$ structure of $\mathcal{A}^{r}$,is an augmented semi-aligned log structure. This yields a morphism

$$
\stackrel{\circ}{\mathcal{A}}^{r} \rightarrow \mathcal{L}_{\log }^{(1 / 2+\varepsilon) \mathrm{al}} .
$$

An aligned log structure determines an augmented semi-aligned log structure (via the trace construction as in (5.2), with trivial cover and isomorphism of trivial covers as augmentation isomorphism). In other words, we have a morphism from $\mathcal{L}_{o g}{ }^{\text {al }}$ to $\log ^{(1 / 2+\varepsilon) a l}$, and this is étale since the stack (5.5) is étale over $\log ^{(1 / 2) a l}$ and the composite

$$
\mathcal{L o g}^{\mathrm{al}} \rightarrow \mathcal{L}^{(1 / 2+\varepsilon) \mathrm{al}} \rightarrow \mathcal{L}^{(1 / 2) \mathrm{al}}
$$

is étale. There is also a morphism

$$
\mathcal{L} \operatorname{ogg}^{(1 / 2+\varepsilon) \mathrm{al}} \rightarrow \stackrel{\circ}{\mathcal{A}}^{1},
$$

mapping an augmented semi-aligned log structure to its degree 2 cover.

Proposition 5.6. The morphisms (5.6) and (5.7) fit into a fiber diagram

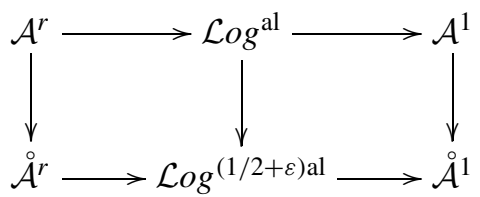

Proof. Since the outer square is a fiber square (Proposition 5.2), it suffices to verify that the right-hand square is a fiber square. This results by observing that upon base change by $\mathcal{A}^{1} \rightarrow \stackrel{\circ}{\mathcal{A}}^{1}$ the composition (5.4) yields a composition of morphisms in (5.3) and applying Corollary 5.5.

Proposition 5.7. Let $X$ be an algebraic stack with locally free log structure $M$, and let $\pi: \widehat{X} \rightarrow X^{\geq 1}$ be a degree 2 finite étale cover with corresponding étale cover $f: F_{\widehat{X} / X} \rightarrow X$ and involution $\iota: F_{\widehat{X} / X} \rightarrow F_{\widehat{X} / X} \cdot$ If $\left(f^{*} M, S^{\prime}\right)$ is an aligned structure with $\iota^{*} S^{\prime}$ related to $S^{\prime}$ by the involution $j$, then there is a unique augmented semi-aligned log structure

$$
\left((M, S), \pi, \widehat{X}^{\geq 2} \rightarrow \mathcal{L}^{\mathrm{al}} \times_{\mathcal{L} \operatorname{og}(1 / 2) \mathrm{al}} X^{\geq 2}\right)
$$

on $X$ such that 
(i) the semi-aligned log structure on $F_{\widehat{X} / X}$ obtained by the trace construction from $S^{\prime}$ is equal to $f^{*} S$, and

(ii) the restriction of $\left(f^{*} M, S^{\prime}\right)$ to $\widehat{X}^{\geq 2}$ is the aligned log structure obtained by projecting from the augmentation isomorphism.

Furthermore, the morphisms corresponding to the log structures fit into a fiber diagram

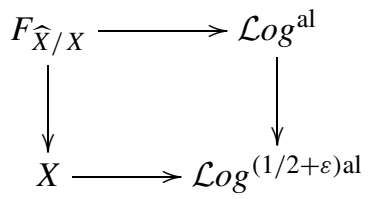

Proof. Condition (i) determines $S$ uniquely, and $(M, S)$ is a semi-aligned log structure on $X$. Restricting $S^{\prime}$, we obtain $\widehat{X} \rightarrow \mathcal{L} \operatorname{og}^{\text {al }}$, and the further restriction to $\widehat{X}^{\geq 2}$ yields the augmentation isomorphism, which is uniquely determined by (ii).

The diagram is 2-commutative. As in the proof of Proposition 5.6, we may reduce the proposition, by adjoining the fiber square with $\mathcal{A}^{1} \rightarrow \stackrel{\circ}{\mathcal{A}}^{1}$ to the right, to the assertion that the outer square of the larger diagram is 2-cartesian. Since the degree 2 cover of the augmented semi-aligned $\log$ structure is $\pi$, we have the assertion by the isomorphism (5.1) and the fact that the construction of Section 5.1 respects base change.

\section{A Local Construction Around a Strictly Semistable Curve}

In this section, we construct an étale neighborhood $\left[\left(X_{g, \triangle}^{\times m}\right)^{\circ} / S_{\mathbf{r}}\right] \rightarrow \mathfrak{M}_{g}^{\text {ss }}$ of any strictly semistable curve $C$ that is stabilizer preserving at a preimage $x^{\prime}$ of $C$ (Proposition 6.1). In order to apply Proposition 3.10 to $\mathfrak{M}_{g}^{\text {ss }}$ (in Section 8), we will need a refinement (Proposition 6.2) that leads to the existence of $U \rightarrow \mathfrak{M}_{g}^{\text {ss }}$, étale and representable, and stabilizer preserving at a preimage $x^{\prime \prime}$ of $C$ (Corollary 6.3).

The morphism $D_{g} \rightarrow \overline{\mathcal{M}}_{g}$ is representable and unramified, and we may consider the algebraic stack $E_{g}=E_{D_{g} / \overline{\mathcal{M}}_{g}}$ (construction of Section 5.1), with étale representable morphism

$$
E_{g} \rightarrow \overline{\mathcal{M}}_{g} .
$$

The stack $E_{g}$ may be viewed as the moduli stack of stable curves of genus $g$ endowed with a choice of at most one node.

\subsection{Stabilization}

We define $X_{g}$ to be the algebraic stack parameterizing semistable curves of genus $g$ with at most one chosen node of the stabilization, that is, the fiber product

$$
X_{g}=\mathfrak{M}_{g}^{\mathrm{ss}} \times \overline{\mathcal{M}}_{g} E_{g} .
$$

We will, by abuse of notation, let st denote also the projection $X_{g} \rightarrow E_{g}$. 
Let $X_{g, \Delta} \subset X_{g}$ be the open substack parameterizing semistable curves $C$ whose stabilization $C \rightarrow C^{\prime}$ has at most one positive-dimensional fiber such that if $c^{\prime} \in C^{\prime}$ has a positive-dimensional fiber, then the node $c^{\prime}$ is chosen.

\subsection{The Local Construction}

Let $C$ be a strictly semistable curve of genus $g$ over an algebraically closed field $k$, and $C^{\prime}$ its stabilization. We attach the following discrete data to $C$. We let $m$ be the number of positive-dimensional fibers of the stabilization $C^{\prime} \rightarrow C, n$ be the maximum number of irreducible components of such a fiber, and $\mathbf{r}=\left(r_{1}, \ldots, r_{n}\right)$ be the sequence of nonnegative integers where $r_{i}$ is the number of such fibers having precisely $i$ irreducible components. Note that $r_{n}>0$ and $\sum_{i} r_{i}=m$; moreover, the number of exceptional components is $\sum_{i} i r_{i}$.

We define

$$
E_{g}^{\times m}=\underbrace{E_{g} \times \overline{\mathcal{M}}_{g} E_{g} \times \overline{\mathcal{M}}_{g} \cdots \times \overline{\mathcal{M}}_{g} E_{g}}_{m \text { times }} .
$$

Similarly, we denote by $X_{g, \triangle}^{\times m}$ the $m$-fold fiber product of $X_{g, \triangle}$ over $\overline{\mathcal{M}}_{g}$. Define $\left(E_{g}^{\times m}\right)^{\circ} \subset E_{g}^{\times m}$ as the open substack parameterizing curves where no pair of selected nodes is the same, and let $\left(X_{g, \triangle}^{\times m}\right)^{\circ}$ be the preimage of $\left(E_{g}^{\times m}\right)^{\circ}$.

The product $S_{\mathbf{r}}=S_{r_{1}} \times \cdots \times S_{r_{n}}$ of symmetric groups acts naturally on the $m$-fold fiber products. Evidently, $\left(E_{g}^{\times m}\right)^{\circ}$ and $\left(X_{g, \triangle}^{\times m}\right)^{\circ}$ are $S_{\mathbf{r}}$-invariant. There is a point $x^{\prime} \in\left(X_{g, \Delta}^{\times m}\right)^{\circ}$ corresponding to the $m$-tuple of semistable curves $\left(C_{1}, \ldots, C_{m}\right)$, where $C_{i}$ is obtained by contracting all exceptional components that do not lie over the $i$ th marked point of the stabilization.

Proposition 6.1. The morphism $\left(X_{g, \triangle}^{\times m}\right)^{\circ} \rightarrow \mathfrak{M}_{g}^{\text {ss }}$, defined by mapping an $m$-tuple $\left(B_{1}, \ldots, B_{m}\right)$ of semistable curves with stabilization $B^{\prime}$ to the fiber product

$$
B_{1} \times{ }_{B^{\prime}} \cdots \times{ }_{B^{\prime}} B_{m},
$$

is étale, representable, and $S_{\mathbf{r}}$-equivariant. Moreover, the induced morphism

$$
\left[\left(X_{g, \Delta}^{\times m}\right)^{\circ} / S_{\mathbf{r}}\right] \rightarrow \mathfrak{M}_{g}^{\mathrm{ss}}
$$

is stabilizer preserving at $x^{\prime}$ and the diagram

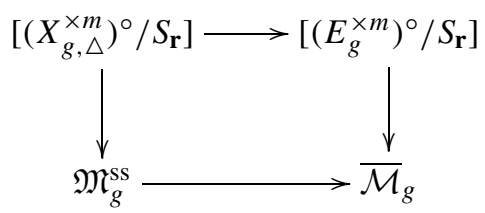

is 2-commutative with étale vertical morphisms and representable induced morphism $\left[\left(X_{g, \triangle}^{\times m}\right)^{\circ} / S_{\mathbf{r}}\right] \rightarrow \mathfrak{M}_{g}^{\mathrm{ss}} \times \overline{\mathcal{M}}_{g}\left[\left(E_{g}^{\times m}\right)^{\circ} / S_{\mathbf{r}}\right]$.

Proof. Since $\left(E_{g}^{\times m}\right)^{\circ} \subset\left(X_{g, \Delta}^{\times m}\right)^{\circ}$ has complement of codimension 2 and $X_{g, \Delta} \rightarrow$ $\overline{\mathcal{M}}_{g}$ is Cohen-Macaulay, $\left(X_{g, \triangle}^{\times m}\right)^{\circ}$ is normal. Since the morphism $\left(X_{g, \triangle}^{\times m}\right)^{\circ} \rightarrow \mathfrak{M}_{g}^{\text {ss }}$ 
is étale in codimension 1, we may conclude from Zariski-Nagata purity [8, Thm. X.3.1] that it is étale. The remaining statements are clear.

Since the morphism $\left[\left(X_{g, \triangle}^{\times m}\right)^{\circ} / S_{\mathbf{r}}\right] \rightarrow \mathfrak{M}_{g}^{\text {ss }}$ is not in general representable (e.g., for $m>1$, the induced morphism on stabilizers is not injective at an $m$-tuple $(B, \ldots, B)$ where $B$ is a smooth curve), some refinement is necessary in order to reduce the verification of Conjecture 1.1 to a stack related to $\left[\left(X_{g, \triangle}^{\times m}\right)^{\circ} / S_{\mathbf{r}}\right]$. We now provide such a refinement.

We recall the degree 2 cover $\widehat{D}_{g} \rightarrow D_{g}$ from Section 2.1. By (5.1) there is a fiber diagram

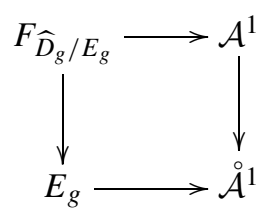

where the bottom arrow is given by $\widehat{D}_{g} \rightarrow D_{g}$. We let $F_{\widehat{D}_{g} / E_{g}}^{\times m}$ be the $m$-fold fiber product over $\overline{\mathcal{M}}_{g}$ and $\left(F_{\widehat{D}_{g} / E_{g}}^{\times m}\right)^{\circ}$ the preimage of $\left(E_{g}^{\times m}\right)^{\circ}$.

Proposition 6.2. There is an étale morphism $[\operatorname{Spec}(A) / H] \rightarrow\left[\left(E_{g}^{\times m}\right)^{\circ} / S_{\mathbf{r}}\right]$, stabilizer preserving at a point $y^{\prime \prime}$ over the image in $\left[\left(E_{g}^{\times m}\right)^{\circ} / S_{\mathbf{r}}\right]$ of $x^{\prime} \in\left(X_{g, \triangle}^{\times m}\right)^{\circ}$ such that the composition

$$
[\operatorname{Spec}(A) / H] \rightarrow\left[\left(E_{g}^{\times m}\right)^{\circ} / S_{\mathbf{r}}\right] \rightarrow \overline{\mathcal{M}}_{g}
$$

is representable. Moreover, there is a finite étale cover $\operatorname{Spec}(B) \rightarrow[\operatorname{Spec}(A) / H]$ such that the composition $\operatorname{Spec}(B) \rightarrow[\operatorname{Spec}(A) / H] \rightarrow\left[\left(E_{g}^{\times m}\right)^{\circ} / S_{\mathbf{r}}\right]$ factors through $\left(F_{\widehat{D}_{g} / E_{g}}^{\times m}\right)^{\circ}$.

Proof. Let $W_{\mathbf{r}}=W_{r_{1}} \times \cdots \times W_{r_{n}}$ be the product of the hyperoctohedral groups of signed permutations. There is an action of $W_{\mathbf{r}}$ on $\left(F_{\widehat{D} / E_{g}}^{\times m}\right)^{\circ}$ compatible with the $S_{\mathbf{r}}$-action on $\left(E_{g}^{\times m}\right)^{\circ}$; this gives an étale morphism $\left[\left(F_{\widehat{D} / E_{g}}^{\times m}\right)^{\circ} / W_{\mathbf{r}}\right] \rightarrow$ $\left[\left(E_{g}^{\times m}\right)^{\circ} / S_{\mathbf{r}}\right]$, which is stabilizer preserving at the unique preimage $\tilde{y}$ in $\left[\left(F_{\widehat{D} / E_{g}}^{\times m}\right)^{\circ} / W_{\mathbf{r}}\right]$ of the image of $x^{\prime} \in\left(X_{g, \triangle}^{\times m}\right)^{\circ}$ in $\left[\left(E_{g}^{\times m}\right)^{\circ} / S_{\mathbf{r}}\right]$. Let $H$ denote the stabilizer group at $\tilde{y}$. By Lemma 3.3 and Section 3.2, there exists an affine scheme $\operatorname{Spec}(A)$ with an action of $H$ and an étale morphism $[\operatorname{Spec}(A) / H] \rightarrow$ $\left[\left(F_{\widehat{D} / E_{g}}^{\times m}\right)^{\circ} / W_{\mathbf{r}}\right]$ stabilizer preserving at a point $y^{\prime \prime} \in[\operatorname{Spec}(A) / H]$ above $\tilde{y}$ such that the composite morphism $[\operatorname{Spec}(A) / H] \rightarrow \overline{\mathcal{M}}_{g}$ is étale and representable.

The final statements are obtained by taking $\operatorname{Spec}(B)$ to be the affine scheme representing the base change $\operatorname{Spec}(A) \times_{\left[\left(F_{\widehat{D} / E_{g}}^{\times m}\right)^{\circ} / W_{\mathbf{r}}\right]}\left(F_{\widehat{D} / E_{g}}^{\times m}\right)^{\circ}$. 
Corollary 6.3. With the notation of Proposition 6.2, if we define $U$ as the fiber product

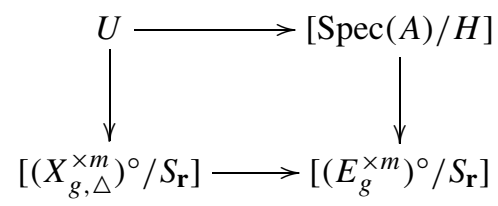

then the composition $U \rightarrow\left[\left(X_{g, \Delta}^{\times m}\right)^{\circ} / S_{\mathbf{r}}\right] \rightarrow \mathfrak{M}_{g}^{\mathrm{ss}}$ is étale and representable, and stabilizer preserving at a preimage $x^{\prime \prime}$ of $C$.

\section{Local Structure of $X_{g}$}

In the section, we exhibit an augmented semi-aligned log structure on $X_{g}$. This semi-aligned $\log$ structure allows us to establish Proposition 7.1, which provides an explicit description of the fiber of the stabilization morphism over a stable curve with precisely one node. This proposition will in turn be applied in Section 8 to complete the proof of Theorem 1.2.

\subsection{Augmented Semi-aligned log Structure on $X_{g}$}

Recall that $X_{g}$ was defined in Section 6.1. We denote by $\widehat{X}_{g}$ the fiber product

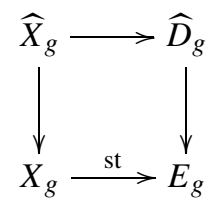

where the degree 2 étale cover $\widehat{D}_{g} \rightarrow D_{g} \subset E_{g}$ is as in Section 2.1. By applying the construction of Section 5.1, diagram (6.1) may be extended to the left with a fiber square

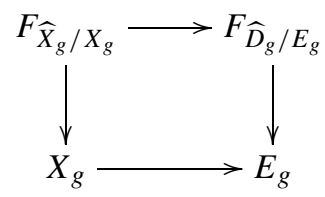

We observe that $F_{\widehat{D}_{g} / E_{g}} \rightarrow \overline{\mathcal{M}}_{g}$ and $\widehat{D}$ satisfy the hypotheses of Proposition 4.1 and thus determine an aligned $\log$ structure on $F_{\widehat{X}_{g} / X_{g}}$. Now Proposition 5.7 may be applied to yield an augmented semi-aligned log structure

$$
X_{g} \rightarrow \mathcal{L} o g^{(1 / 2+\varepsilon) \mathrm{al}}
$$


fitting into a fiber diagram

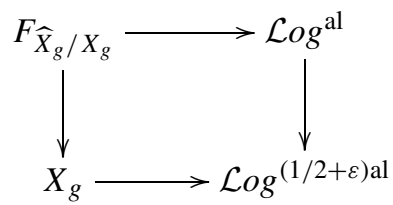

with the morphism corresponding to the aligned log structure on $F_{\widehat{X}_{g} / X_{g}}$ and vertical étale morphisms.

\subsection{Structure of $X_{g, \Delta}$}

The next result is an analogue of Lemma 4.3 for $X_{g, \Delta}$.

Proposition 7.1. There is a fiber diagram

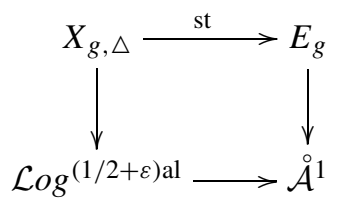

where the left vertical arrow is the restriction to $X_{g, \Delta}$ of the morphism (7.2), and the right vertical arrow is given by the finite étale cover $\widehat{D}_{g} \rightarrow D_{g}$.

Proof. Since the morphisms $X_{g, \Delta} \rightarrow E_{g}$ and $\log ^{(1 / 2+\varepsilon) \text { al }} \rightarrow \stackrel{\circ}{\mathcal{A}}^{1}$ are isomorphisms in codimension 1 , so is the morphism

$$
\Psi: X_{g, \Delta} \rightarrow \mathcal{L} \log ^{(1 / 2+\varepsilon) \mathrm{al}} \times{\stackrel{\circ}{\mathcal{A}^{1}}} E_{g} .
$$

The fiber product in (7.5) is smooth (since the right-hand morphism in (7.4) is smooth), so it follows from the Zariski-Nagata purity [8, Thm. X.3.1] that $\Psi$ is étale. Since $\Psi$ is also bijective on geometric points and is pointwise stabilizer preserving, we conclude from Lemma 3.1 that $\Psi$ is an isomorphism.

\section{Proof of Theorem 1.2}

THEOREM 8.1. Conjecture 1.1 holds for $\mathfrak{M}_{g}^{\mathrm{ss}}$.

Proof. Let $C$ be a strictly semistable curve with stabilization $C^{\prime}$. Let $m$ and $\mathbf{r}=$ $\left(r_{1}, \ldots, r_{n}\right)$ be the combinatorial data assigned to $C$ as introduced in Section 6.2.

We apply Proposition 6.2 and, with the notation from there, define

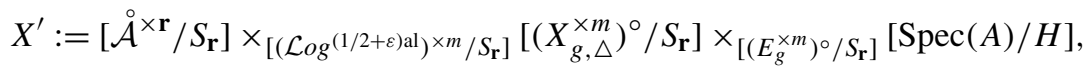

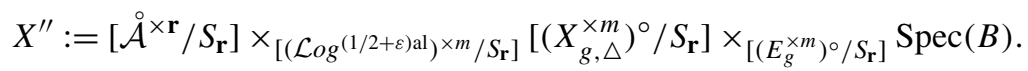

Notice, with the notation of Corollary 6.3, that $X^{\prime}$ may be identified with the fiber product $\left[\mathcal{A}^{\times \mathbf{r}} / S_{\mathbf{r}}\right] \times{ }_{\left[\left(\mathcal{L}_{\log }^{(1 / 2+\varepsilon) a l}\right)^{\times m} / S_{\mathbf{r}}\right]} U$, and hence (cf. Proposition 5.6) admits 
a morphism to $\mathfrak{M}_{g}^{\text {ss }}$ satisfying conditions (iii) and (iv) of Proposition 3.10. The cover $X^{\prime \prime} \rightarrow X^{\prime}$ satisfies condition (ii). So it remains to verify condition (i).

By Proposition 6.2, $X^{\prime \prime}$ is isomorphic to

$$
\left.\stackrel{\circ}{\mathcal{A}} \times \mathbf{r} \times_{\left(\mathcal{L}_{o g}(1 / 2+\varepsilon) \mathrm{al}\right) \times m}\left(F_{\widehat{X}_{g, \Delta} \times m}^{\times m}\right)_{g, \Delta}\right)^{\circ} \times_{\left(F_{\widehat{D}_{g} / E_{g}}^{\times m}\right)^{\circ}} \operatorname{Spec}(B),
$$

where $\widehat{X}_{g, \Delta}$ denotes the preimage of $X_{g, \Delta}$ under $\widehat{X}_{g} \rightarrow X_{g}$, and where $\left(F_{\widehat{X}_{g, \Delta} / X_{g, \Delta}}\right)^{\circ}$ denotes the preimage of $\left(E_{g}^{\times m}\right)^{\circ}$ in the $m$-fold fiber product $F_{\widehat{X}_{g, \Delta} \times X_{g, \Delta}}^{\times m}$ over $\overline{\mathcal{M}}_{g}$. Thanks to (7.3) and Proposition 5.6, $X^{\prime \prime}$ may be identified with

$$
\left.\mathcal{A}^{\times \mathbf{r}} \times_{\left(\mathcal{L}_{o g} \mathrm{al}\right)^{\times m}}\left(F_{\widehat{X}_{g, \Delta} / X_{g, \Delta}}^{\times m}\right)^{\circ} \times_{\left(F_{\widehat{D}_{g} / E_{g}}^{\times m}\right.}\right)^{\circ} \operatorname{Spec}(B) .
$$

The next step is to obtain from diagrams (6.1), (7.1), and (7.3) and from Propositions 5.6 and 7.1 the fiber diagram

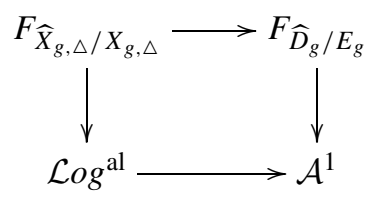

This yields further fiber diagrams upon passing to $m$-fold fiber products (in the top row, over $\left.\overline{\mathcal{M}}_{g}\right)$ and upon restricting to preimages of $\left(E_{g}^{\times m}\right)^{\circ}$. Now we have

$$
X^{\prime \prime} \cong \mathcal{A}^{\times \mathbf{r}} \times{ }_{\left(\mathcal{A}^{1}\right) \times m} \operatorname{Spec}(B),
$$

and we conclude, as in the proof of Theorem 4.2, by observing that the morphism from $\operatorname{Spec}(B)$ to $\left(\mathcal{A}^{1}\right)^{\times m}$ is affine.

ACKNOWLEDGMENTS. We are grateful for the valuable suggestions provided by the referee. We thank Rahul Pandharipande for stimulating discussions that motivated this investigation. We also thank the referee for useful comments and Jack Hall for providing comments on the first draft.

\section{References}

[1] D. Abramovich, C. Cadman, B. Fantechi, and J. Wise, Expanded degenerations and pairs, Comm. Algebra 41 (2013), no. 6, 2346-2386.

[2] D. Abramovich, M. Olsson, and A. Vistoli, Tame stacks in positive characteristic, Ann. Inst. Fourier (Grenoble) 58 (2008), no. 4, 1057-1091.

[3] J. Alper, On the local quotient structure of Artin stacks, J. Pure Appl. Algebra 214 (2010), no. 9, 1576-1591.

[4] E. Arbarello, M. Cornalba, and P. A. Griffiths, Geometry of algebraic curves, Vol. II, Springer, Heidelberg, 2011.

[5] B. Conrad and A. J. de Jong, Approximation of versal deformations, J. Algebra 255 (2002), no. 2, 489-515.

[6] P. Deligne and D. Mumford, The irreducibility of the space of curves of given genus, Inst. Hautes Études Sci. Publ. Math. 36 (1969), 75-109.

[7] D. Edidin, B. Hassett, A. Kresch, and A. Vistoli, Brauer groups and quotient stacks, Amer. J. Math. 123 (2001), no. 4, 761-777. 
[8] A. Grothendieck, Revêtements étales et groupe fondamental (SGA 1), Lecture Notes in Math., 224, Springer-Verlag, Berlin, 1971.

[9] C. Huneke, Complete ideals in two-dimensional regular local rings, Commutative algebra (Berkeley, CA, 1987), Math. Sci. Res. Inst. Publ., 15, pp. 325-338, Springer, New York, 1989.

[10] S. Keel and S. Mori, Quotients by groupoids, Ann. of Math. (2) 145 (1997), no. 1, 193-213.

[11] A. Kresch, Flattening stratification and the stack of partial stabilizations of prestable curves, Bull. Lond. Math. Soc. 45 (2013), no. 1, 93-102.

[12] G. Laumon and L. Moret-Bailly, Champs algébriques, Springer-Verlag, Berlin, 2000.

[13] D. Luna, Slices étales, Bull. Soc. Math. France, Mém., 33, pp. 81-105, Soc. Math. France, Paris, 1973.

[14] A. M. Mustaţă and A. Mustaţă, The structure of a local embedding and Chern classes of weighted blow-ups, J. Eur. Math. Soc. 14 (2012), no. 6, 1739-1794.

[15] M. C. Olsson, Logarithmic geometry and algebraic stacks, Ann. Sci. École Norm. Sup. (4) 36 (2003), no. 5, 747-791.

[16] D. Oprea, Tautological classes on the moduli spaces of stable maps to $\mathbb{P}^{r}$ via torus actions, Adv. Math. 207 (2006), no. 2, 661-690.

[17] D. Rydh, The canonical embedding of an unramified morphism in an étale morphism, Math. Z. 268 (2011), no. 3-4, 707-723.

[18] Existence and properties of geometric quotients, J. Algebraic Geom. 22 (2013), no. 4, 629-669.

[19] , Noetherian approximation of algebraic spaces and stacks, J. Algebra 422 (2015), 105-147.

[20] H. Sumihiro, Equivariant completion, II, J. Math. Kyoto Univ. 15 (1975), no. 3, 573605.

[21] The Stacks Project authors, Stacks Project, 〈http://stacks.math.columbia.edu〉.

J. Alper

Mathematical Sciences Institute

Australia National University

Canberra

ACT 0200

Australia

Institut Für Mathematik

Humboldt-Universität zu Berlin

Unter den Linden 6

10099 Berlin

Germany
A. Kresch

Institut für Mathematik

Universität Zürich

Winterthurerstrasse 190

CH-8057 Zürich

Switzerland

andrew.kresch@math.uzh.ch

jarod.alper@math.anu.edu.au 